\title{
GENERAL RELATIVITY, PARTIAL DIFFERENTIAL EQUATIONS, AND DYNAMICAL SYSTEMS
}

\author{
ARTHUR E. FISCHER AND JERROLD E. MARSDEN
}

0 . Introduction. In this paper we study two aspects of the Einstein equations of evolution for an empty spacetime. In the first part (\$\$1-3) we give a simple direct proof that the differentiability of the Cauchy data is maintained for short time. In the second part ( $\$ \$ 4-5)$ we sketch how, on a suitable configuration space, Einstein's equations can be considered as forced geodesics modified by terms which reflect a moving coordinate system equipped with its own system of clocks. Both of these topics will be presented in more detail elsewhere [15], [12].

Let $\Omega$ be a bounded open domain in $R^{3}$, let $g_{\mu v}\left(x^{\prime}\right)$. $\left(x^{\prime}\right) \in \Omega \subset \mathbf{R}^{3}, 0 \leqq \mu, v \leqq 3$. $1 \leqq i \leqq 3$, be a Lorentz metric of signature $(-,+,+,+)$, and let $k_{\mu v}\left(x^{\prime}\right)$ be a symmetric 2-covariant tensor field on $\Omega$. The first proof that Cauchy data $\left(\xi_{u v}\left(x^{i}\right), k_{\mu v}\left(x^{i}\right)\right)$ of Sobolev class $\left(H^{3}, H^{s-1}\right), s \geqq 4$, evolves for small time into a Ricci zero $\left(R_{\mu \nu}=0\right)$ spacetime $g_{\mu \nu}\left(t, x^{\prime}\right)$ which is also of class $\boldsymbol{H}^{s}$ was given by Choquel-Bruhat [3], [4], based on earlier work by herself [2], and Lichnerowicz [21]. Her method of proof is to normalize the Ricci tensor by using harmonic coordinates so that the resulting system is a quasilinear strictly hyperbolic system (no multiple characteristics). The result then follows by quoting a theorem of Leray [20. p. 230] about quasilinear strictly hyperbolic systems, as modified by Dionne [7. p. 82]. Leray's original version of the theorem loses a derivative (i.e., $H^{3}$ Cauchy data only has an $H^{s-1}$ evolution), but Dionne remedies this defect.

Our method of proof is based on a simple observation; namely, the Ricci tensor in harmonic coordinates can be reduced to a quasilinear symmetric hyperbolic first order system of the form

$$
A^{0}(u) \partial u / \partial t=A^{\prime}(u) \partial u / \partial x^{\prime}+B(u) \text {. }
$$

where $u$ and $B(u)$ are 50 component column vectors, $A^{\circ}(u)$ and $A^{\prime}(u)$ are symmetric,

AMS 1970 subjert classifications. Primary 83C 99, 83C10. 35L45. 35L60: Secondary 83C30, 83C20, 83C45. 35LOS. 
and $A^{\circ}(u)$ is positive-definite. This observation is inspired by the well-known facl that any single second order hyperbolic equation can be reduced to a first order symmetric hyperbolic system.

Let $g_{x \rho}(t, x)$ be an $H^{3}$-solution of $R_{a v}=0$ with given Cauchy data. Because of the form invariance of the system $R_{\mu \nu}=0 . \bar{g}_{\mu v}=\left(\partial x^{2} / \partial \bar{x}^{\mu}\right)\left(\bar{c} x^{\beta} / \hat{r} \bar{x}^{\prime \prime}\right) g_{2 a}$ is also an $H^{3}$-solution of $R_{\mu v}=0$, if $\bar{x}^{a}\left(x^{\prime \prime}\right)$ is an $H^{s+1}$-coordinate transformation. By arranging so that $\bar{x}^{3}\left(x^{\mu}\right)$ is the identity in a neighborhood of the spacelike hypersurface $t=0, \bar{g}_{u,}$ has the same Cauchy data as $g_{u r}$. Hence solutions to the Cauchy problem cannot be functionally unique. However. we prove a uniqueness theorem that says the evolution is unique up to the $H^{x+1}$-isometry class of the spacetime. This result sharpens, by one degree of differentiability. the uniqueness theorem stated in [3].

In [15] we shall show how our existence and uniqueness theorems can be obtained intrinsically for arbitrary manifolds. not necessarily compact, in the class of metrics for which the space-manifold is complete and which satisfies suitable asymptotic conditions.

In $\$ \$ 4-5$ we consider in what sense Einstein's equations in 3-dimensional form are a Lagrangian system of the classical form kinetic energy minus potential energy. We show how on a suitable configuration space (the manifold $/ \times$. $/ 1$ ). the evolution equations are a degenerate dynamical system. Various terms in the Einstein system are given a geometrical explanation. In particular, the central role played by certain Lie derivative terms in the presence of a shift vector field is shown to be analogous to the space-body transitions of hydrodynamics (see Ebin-Marsden [9]) or the rigid body (see Marsden-Abraham [23]). These results are a geometrical reinterpretation of the basic work of Arnowitt-Deser-Misner [1]. DeWitt [6] and Wheeler [29].

We thank D. Ebin, H. Lewy, P. Lax, M. Protter, R. Sachs, and A. Taub for a variety of helpful suggestions.

1. Existence of an $H^{3}$-spacetime for $\left(H^{s}, H^{s-1}\right)$ Cauchy data. For emply space relativity, one searches for a Lorentz metric $g_{\mu v}\left(l, x^{i}\right)$ whose Ricci curvature $R_{\mu v}$ is zero: i.e., $g_{\mu v}\left(1, x^{i}\right)$ must satisfy the system

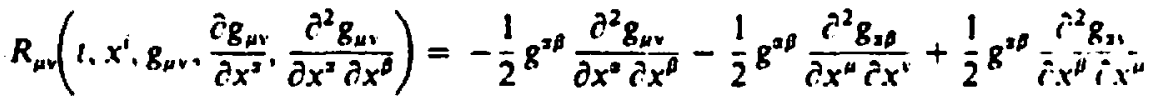

$$
\begin{aligned}
& +\frac{1}{2} g^{2 \phi} \frac{\partial^{2} g_{z \mu}}{\partial x^{\beta} \partial x^{v}}+H_{\mu v}\left(g_{\mu v \cdot} \frac{\partial g_{\mu v}}{\partial x^{2}}\right) \\
& =0
\end{aligned}
$$

where $H_{\mu v}\left(g_{u v}, \partial_{g u v} / \partial x^{a}\right)$ is a rational combination of $g_{\mu v}$ and $\gamma_{u \mu} i x^{2}$ with denominator det $g_{\mu v} \neq 0$. Note that the contravariant tensor $g^{\mu r}$ is a tational combination of the $g_{\mu v}$ 's with denominator det $g_{\mu v} \neq 0$. 
Let $G_{\mu \nu}=R_{\mu v}-\frac{1}{2} g_{\mu \nu} R$ be the Einstein tensor, where $R=g^{x g} R_{\alpha \rho}$ is the scalar

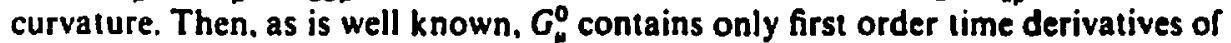
$g_{\mu v}$. Thus $G_{\mu}^{\prime \prime}\left(0, x^{\prime}\right)$ can be computed from the Cauchy data $g_{\mu v}\left(0, x^{\prime}\right)$ and $\partial g_{\mu \nu}\left(0, x^{\prime}\right) / \partial t$ alone, and therefore $G_{\mu}^{U}\left(0, x^{i}\right)=0$ is a necessary condition on the Cauchy data in order that a spacetime $g_{\mu v}\left(1, x^{\prime}\right)$ have the given Cauchy data and satisfy $G_{\mu v}=0$, which is equivalent to $R_{\mu v}=0$.

The existence part of the Cauchy problem for the system $R_{\mu v}=0$ is as follows:

Let $\left(\dot{g}_{\mu v}\left(x^{i}\right), \hat{k}_{\mu v}\left(x^{i}\right)\right)$ be Cauchy data of class $\left(H^{\prime}(\Omega), H^{s-1}(\Omega)\right), s \geqq 4$, such that $\mathcal{G}_{\mu}^{0}\left(x^{i}\right)=0$. Let $\Omega_{0}$ be a proper subdomain, $\bar{\Omega}_{0} \subset \Omega$. Find an $\varepsilon>0$ and a spacetime $g_{u v}\left(t, x^{i}\right),|t|<\varepsilon_{0}\left(x^{i}\right) \in \Omega_{0} \subset \Omega$ such that

(a) $g_{u v}\left(t, x^{\prime}\right)$ is $H^{\prime}$ joint $\left(y\right.$ in $\left(\ell, x^{i}\right) \in(-\varepsilon, \varepsilon) \times \Omega_{0}$.

(b) $\left(g_{\mu v}\left(0, x^{\prime}\right), \partial g_{\mu v}\left(0, x^{\prime}\right) / \partial t\right)=\left(g_{\mu v}\left(x^{\prime}\right), k_{u v}\left(x^{\prime}\right)\right)$, and

(c) $B_{u v}\left(t, x^{\prime}\right)$ has zero Ricci curvalure.

The system $R_{u v}=0$ is a quasilinear system of ten second order partial difierential equations for which the highest order terms involve mixing of the components of the system. As it stands, there are no known theorems about partial differential equations which can be applied to resolve the Cauchy problem. However, as was first noted in 1922 by Lanczos [18] (and in fact in 1916 by Einstein himself for the linearized equations [10]) the Ricci tensor simplifies considerably in harmonic coordinates, i.e., in a coordinate system $\left(x^{a}\right)$ for which the contracted Christoffel symbols vanish, $\Gamma^{\mu}=g^{\alpha A} \Gamma_{\alpha \beta}^{\mu}=0$. (For the existence of such a coordinate system for an arbitrary Lorentz melric see Theorem 3.3.) In fact, an algebraic computation shows that

$$
R_{\mu v}=-\frac{1}{2} g^{\alpha \beta} \frac{\partial^{2} g_{\mu v}}{\partial x^{2} \partial x^{\phi}}+\frac{1}{2} g_{\mu s} \frac{\partial \Gamma^{\alpha}}{\partial x^{v}}+\frac{1}{2} g_{v z} \frac{\partial \Gamma^{\nu}}{\partial x^{\mu}}+H_{\mu v}
$$

so that in a coordinate system for which $\Gamma^{\mu}=0$,

$$
R_{\mu v}=R_{\mu v}^{\left(h_{v}\right)}=-\frac{1}{2} g^{a \theta}\left(\partial^{2} g_{\mu v} / \partial x^{a} \partial x^{\rho}\right)+H_{\mu v}
$$

The operator $-\frac{1}{2 g^{\alpha B}}\left(\partial^{2} / \partial x^{a} \partial x^{\beta}\right)$ operates the same way on each component of the system $g_{u v}$ so that there is no mixing in the highest order derivatives. Thus the normalized system $R_{\mu v}^{(h)}=0$ is considerably simpler than the full system. In fact, the system $R_{\mu \nu}^{(h)}=0$ has only simple characteristics so that $R_{\mu v}^{(h)}=0$ is a strictly hyperbolic system.

The importance of the use of harmonic coordinates and of the system $R_{\mu v}^{(t)}=0$ is based on the fact that it is sufficient to solve the Cauchy problem for $R_{\mu v}^{(\mathrm{h})}=0$; this remarkable fact discovered by Foures-Bruhat [2] is based on the observation that the condition $\Gamma^{\mu}\left(x^{j}\right) \equiv \dot{g}^{2 \theta}\left(x^{j}\right) \Gamma^{\mu}{ }_{p}\left(x^{i}\right)=0$ is propagated off the hypersurface $t=0$ for solutions $g_{u v}$ of $R_{\mu v}^{(h)}=0$. This is established in the next lemma.

1.1. LEMMA. Let $\left(\xi_{\mu v}\left(x^{\prime}\right), k_{\mu v}\left(x^{\prime}\right)\right)$ be of Sobolev class $\left(H^{\prime}, H^{z-1}\right)$ on $\Omega, s>\frac{1}{2} n+2$, $n=3$, and suppose that $\left(\xi_{u v}\left(x^{\prime}\right), \dot{k}_{u v}\left(x^{\prime}\right)\right)$ sarisfies 
(a) $T^{\mu}\left(x^{3}\right)=0$

(b) $G_{u}^{0}\left(x^{i}\right)=0$.

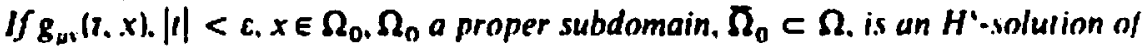

$$
\begin{aligned}
& \left(g_{\mu v}(0, x), \hat{c} g_{\mu v}(0, x) / \partial t\right)=\left(\xi_{u v}\left(x^{i}\right), k_{\mu v}\left(x^{i}\right)\right) . \\
& R_{\mu v}^{(h)}=-\frac{1}{2} g^{2 \theta}\left(r^{2} g_{u v} / \hat{r} x^{2} \partial x^{b}\right)+H_{\mu v}=0 \text {. }
\end{aligned}
$$

then $\Gamma^{\mu}\left(t, x^{i}\right)=0$ for $|r|<\varepsilon, x \in \Omega_{0}$.

Proof. The case $s>\frac{1}{2} n+2$ is treated in [14]: here we assume $s>1 n+3$. Let $g_{\mu v}\left(t, x^{\prime}\right)$ satisfy (a), (b) and $R_{u v}^{(h)}=0$. Then a straightforward computation shows that $\Gamma^{\mu}\left(t, x^{\prime}\right)=g^{\alpha \beta}\left(t, x^{\prime}\right) \Gamma_{\alpha \beta}^{\mu}\left(t, x^{\prime}\right)$ satisfies $\partial \Gamma^{\mu}\left(0, x^{i}\right) / \lambda t=0$. From $G^{\mu \prime}{ }_{i v}=0$ (where "means covariant derivative) and $R_{\mu \nu}^{(h)}=0, \Gamma^{\mu}$ is shown to satisfy' the system of linear equations

$$
g^{\alpha \beta} \frac{\partial^{2} \Gamma^{\mu}}{\partial x^{\alpha} \partial x^{\beta}}+A_{a}^{\alpha \mu}\left(g_{\mu v} \cdot g^{\mu \nu} \cdot \frac{\partial g_{\mu v}}{\partial x^{\alpha}}\right) \frac{\partial \Gamma^{\alpha}}{\partial x^{\beta}}=0 .
$$

This linear system can be reduced to a linear first order symmetric hyperholic system for which a uniqueness and existence theorem holds. This is exacily analngous to Theorems 1.2 and 3.1 below. But from the uniqueness for this system. $\Gamma^{\mu}\left(0, x^{i}\right)=0$ and $\partial \Gamma^{\mu}\left(0, x^{i}\right) / \partial t=0$ imply $\Gamma^{\mu \prime}\left(t, x^{i}\right)=0$.

According to the lemma, an $H^{s}$-solution of $R_{\mu v}^{(h)}=0$ with prescribed Cuuch! data is also a solution of $R_{\mu \nu}=0$ (since $\Gamma^{\mu}(1, x)=0 \Rightarrow R_{\mu v}^{(h)}=R_{\mu v}$ ). provided that the Cauchy data satisfies (a) $\Gamma^{\mu}=0$ and (b) $C_{\mu}^{0}=0$. As mentioned above $(b)$ is a necessary condition on the Cauchy data for a solution $g_{\mu v}(t, x)$ to satisly $R_{u}=0$. If $(a)$ is not satisfied, then a set of Cauchy data can be found whose evolution under $R_{u r}^{(t)}=0$ leads to an $H^{s}$-spacetime which by an $H^{+1}$-coordinate transformation gives rise to a spacetime with the original Cauchy data (see Fischer-Marsden [14]).

From the theorem of Dionne concerning quasilinear strictly hyperbolic system. [7] and Lemma 1.1, Choquet-Bruhat [3]. [4] concludes that Cauchy data of class $\left(H^{s} . H^{s-1}\right)$ has an $H^{s}$-time evolution. We prove this result directly by reducing the strictly hyperbolic system $R_{u v}^{(h)}=0$ to a quasilinear symmetric hyperbolic first order system. Aside from the practical nature of putting the Einstein evolution equations in this form (systems of this type are very well understood: the Cauchy problem has a simple resolution in this form), there is the aesthetic value of bringing relativity into a form which uniformly governs most other equations of mathematical physics, such as Maxwell's equations, the Dirac equation, the Lundquist equations of magnetohydrodynamics. Euler's equations for a compressible fiuid. and the equations describing the motion of elastic bodies.

1.2. THEOREM. Let $\Omega$ be an open bounded domain in $\mathbf{R}^{3}$ with $\Omega_{0}$ a proper suhclumuin. $\Omega_{0} \subset \Omega$, and let $\left(\xi_{\mu v}(x), \dot{k}_{\mu v}(x)\right),\left(x^{\prime}\right) \in \Omega, 0 \leqq \mu, v \leqq 3.1 \leqq i \leqq 3$, be of Sobolev class 
$\left(H^{3}, H^{s-1}\right), s \geqq 4$. Suppose that $\Gamma^{\prime \prime}\left(x^{\prime}\right)=0$ and $G_{\mu}^{0}(x)=0$. Then there exists an $\varepsilon>0$ and $a$ unique Lorent $z$ metric $g_{\mu v}(t, x), \mid t_{1}^{\prime}<\varepsilon_{1}\left(x^{\prime}\right) \in \Omega_{0}$ such that

(1) $g_{u v}\left(t, x^{i}\right)$ is jointly of class $H^{3}$,

(2) $R_{\mu v}^{(h)}\left(1, x^{i}\right)=0$.

(3) $\left(g_{\mu v}\left(0, x^{\prime}\right), \partial g_{\mu v}\left(0, x^{\prime}\right) / \partial t\right)=\left(g_{\mu v}\left(x^{\prime}\right), k_{\mu v}\left(x^{\prime}\right)\right)$.

From Lemma 1.1, this $g_{\mu v}\left(t, x^{\prime}\right)$ also satisfies $R_{\mu v}\left(l, x^{\prime}\right)=0$. Moreoter, $g_{u v}\left(t, x^{\prime}\right)$ depends continuously on $\left(\xi_{u v}\left(x^{\prime}\right), k_{u v}\left(x^{\prime}\right)\right)$ in the $\left(H^{3}, H^{-1}\right)$ sopology. If $\left(\xi_{u v}\left(x^{\prime}\right)\right.$, $\left.k_{\mu v}\left(x^{i}\right)\right)$ is of class $\left(C^{\infty}, C^{\infty}\right)$ on $\Omega$, then $g_{\mu v}\left(t, x^{\prime}\right)$ is $C^{\infty}$ for all $i$ for which the solution exists.

Note. The case $s=4$ is delicate and is treated in [14]. Here we assume $s \geqq 5$. In [14] we also give a complete discussion for the case of spatial asymptotic conditions.

PROOF. The system $R_{\mu v}^{(t)}=0$ is reduced to a first order system by introducing the isn new unknowns $k_{\mu v}=\partial g_{\mu v} / \partial t$ and the thirty new unknowns $g_{\mu v, l}=\partial g_{\mu v} / \partial x^{\prime}$ a 1 il considering the quasilinear first order system of fifty equations:

(Q)

$$
\begin{aligned}
& \hat{c} g_{u v} / \partial t=k_{\mu v} \text {. } \\
& g^{i j}\left(\partial g_{\mu v, d} / \partial t\right)=g^{j j} \frac{\partial k_{\mu v}}{\partial x^{i}} \\
& -g^{00} \frac{\partial k_{\mu v}}{\partial t}=2 g^{0 J} \frac{\partial k_{\mu v}}{\partial x^{j}}+g^{\prime \prime} \frac{\partial g_{\mu v, J}}{\partial x^{J}}-2 H_{\mu v}\left(g_{\mu v}, g_{\mu v, l}, k_{\mu v}\right) \text {. }
\end{aligned}
$$

We are considering $H_{\mu v}$ as a polynomial in $g_{\mu v, j}$ and $k_{\mu v}$ and rational in $g_{\mu v}$ with denominator det $g_{\mu v} \neq 0$. At first, we extend our initial data to all of $\mathbf{R}^{3}$, say to equal the Minkowski metric outside a compact set, and consider the system (Q) on $\mathbf{R}^{3}$. Note that the Cauchy data need not satisfy the constraints $G_{\mu}^{0}=0$ during the transition.

The matrix $g^{i j}$ has inverse $g_{j k}-\left(g_{j 0} g_{k 0} / g_{00}\right)\left(i . e_{.} g^{\prime \prime}\left(g_{j k}-\left(g_{j 0} g_{k 0} / g_{00}\right)\right)=\delta_{k}^{\prime}\right)$ so that the second set of thirty equations can be inverted to give

$$
\partial g_{\mu v, d} / \partial t=\partial k_{\mu v} / \partial x^{l}
$$

For $g_{\mu v}$ of class $C^{2},(1)$ implies

$$
g_{\mu v, i}=\partial g_{\mu v} / \partial x^{i}
$$

so that the system (Q) is equivalent to $R_{\mu \nu}^{(h)}=0$.

Let

$$
u=\left(\begin{array}{l}
B_{\mu v} \\
g_{\mu v, l} \\
k_{\mu v}
\end{array}\right)
$$


be a fift! component column vector, where $g_{u}$, , is listed as

$$
\text { - }\left(\begin{array}{c}
g_{00.1} \\
\vdots \\
g_{33.1} \\
\vdots \\
800.3 \\
\vdots \\
8_{33.3}
\end{array}\right) .
$$

$0^{10}=10 \times 10$ zero matrix, $I^{10}=10 \times 10$ identity matrix, and let $A^{\prime \prime}(u)=$ $A^{0}\left(g_{\mu v}, g_{\mu v, j}, k_{\mu v}\right)$ and $A^{j}\left(g_{\mu v}, g_{\mu v, 1}, k_{\mu \nu}\right)$ be the $50 \times 50$ matrices given by

$$
\begin{aligned}
& A^{0}\left(g_{\mu v} \cdot g_{\mu v .1} \cdot k_{\mu v}\right)=\left(\begin{array}{ccccc}
f^{10} & 0^{10} & 0^{10} & 0^{10} & 0^{11} \\
0^{10} & g^{11} /^{10} & g^{12} /^{10} & g^{13} /^{10} & 0^{10} \\
0^{10} & g^{12} f^{10} & g^{22} /^{10} & g^{23} /^{10} & 0^{10} \\
0^{10} & g^{13} /^{10} & g^{23} /^{10} & g^{33} /^{10} & 0^{10} \\
0^{10} & 0^{10} & 0^{10} & 0^{10} & -g^{10} / 10
\end{array}\right) . \\
& A^{j}\left(g_{\mu v} \cdot g_{u v .1} \cdot k_{\mu v}\right)=\left(\begin{array}{ccccc}
0^{10} & 0^{10} & 0^{10} & 0^{10} & 0^{10} \\
0^{10} & 0^{10} & 0^{10} & 0^{10} & g^{j 1} J^{10} \\
0^{10} & 0^{10} & 0^{10} & 0^{10} & g^{j 2} J^{10} \\
0^{10} & 0^{10} & 0^{10} & 0^{10} & g^{j 3} /^{10} \\
0^{10} & g^{1 / J^{10}} & g^{2 / J^{10}} & g^{3 / J^{10}} & 2 g^{j 0} f^{10}
\end{array}\right) .
\end{aligned}
$$

and let $B\left(g_{u v}, g_{\mu v, 1}, k_{\mu v}\right)$ be the fifiy component column vector given by

$$
B\left(g_{\mu v}, g_{\mu v, j,}, k_{\mu v}\right)=\left(\begin{array}{c}
k_{\mu v} \\
0^{30} \\
-2 H_{\mu v}\left(g_{\mu v}, g_{u v, i}, k_{u v}\right)
\end{array}\right)
$$

where $0^{30}$ is the thirty component zero column vector.

Note that $A^{0}(u)$ and $A^{j}(u)$ are symmetric, and that $A^{0}(u)$ is positive-definite if $g_{\mu v}$ has Lorentz signature. A direct verification shows that the first-order quasilinear symmetric hyperbolic system 


$$
A^{0}(u)(\partial u / \partial t)=A^{j}(u)\left(\partial u / \partial x^{j}\right)+B(u)
$$

is just the system (Q). From Theorem 2.1 and its generalizations proven below, we conclude that for Cauchy data

$$
u\left(x^{i}\right)=\left(\begin{array}{l}
\xi_{u v}\left(x^{\prime}\right) \\
\xi_{u v, 0}\left(x^{\prime}\right) \\
k_{u v}\left(x^{\prime}\right)
\end{array}\right)
$$

of Sobolev class $H^{s-1}, s-1>\frac{1 n}{2}+2$, there exists an $\varepsilon>0$ and a solution

$$
u\left(t, x^{i}\right)=\left(\begin{array}{l}
g_{u v}\left(t, x^{\prime}\right) \\
g_{u v, j}\left(1, x^{\prime}\right) \\
k_{u v}\left(1, x^{i}\right)
\end{array}\right)
$$

of class $H^{s-1}$. By Sobolev's lemma, $u\left(\ell, x^{i}\right)$ is also of class $C^{2}$, and so, by the second set of equations of $\left(Q h g_{\mu v, i}=\partial g_{\mu v} / \partial x^{\prime}\right.$. Since $\left(g_{\mu v, i v} k_{\mu v}\right)=\left(\partial g_{\mu v} / \partial x^{\prime}, \partial g_{\mu v} / \partial t\right)$ is of class $H^{s-1} \cdot g_{u v}\left(1, x^{i}\right)$ is in fact of class $H^{3}$. The continuous dependence of the solutions on the initial data follows from the general theory below.

To recover the result for the domain $\Omega$ from the result for $\mathbf{R}^{n}$, we can use the standard domain of dependence arguments; see Courant-Hilbert [5].

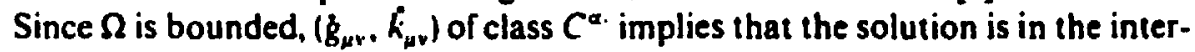
section of all the Sobolev spaces and hence is $C^{\alpha}$; again we are using a general regularity result about symmetric hyperbolic systems.

From Lemma 1.1 , the $\boldsymbol{g}_{\mu v}\left(t, x^{i}\right)$ so found satisfy the field equations $R_{\mu v}=0$. For the case in which the Cauchy data does not satisly $f^{\prime \prime}\left(x^{\prime}\right)=0$, see [14].

Although $g_{u v}\left(f, x^{\prime}\right)$ is a unique solution of $R_{u v}^{(h)}=0$, with prescribed Cauchy data. it is not a unique solution of $R_{\mu v}=0$. We return to this point in $\$ 3$.

2. First order quasilinear symmetric hyperbolic systems. The theory of linear first order symmetric hyperbolic systems is due to Friedrichs [17] with some simplifying modifications by Lax [19]. The essential ideas for handling quasilinear equations appear in Schauder [25]. Frankl [16]. and Petrovskii [24]. Friedrichs [17. p. 352] mentions that these ideas can be used to prove the unique existence of a solution for a quasilinear first order symmetric hyperbolic system. and it is again mentioned in Courant-Hilbert [5, p. 675], but we have been unable to find the details of a complete treatment in the literature. Here we shall outline the methods for $\mathbf{R}^{n}$ and present an intrinsic version for manifolds elsewhere [15]. The basic idea is to find energy type estimates, use the contraction mapping principle to find an $H^{3-1}$-solution, and then show that this $H^{3-1}$-solution is in fact $H^{2}$.

2.1. Theorem. Let $H^{3}\left(\mathbf{R}^{n}, \mathbf{R}^{m}\right)$ denote the $H^{s}$ maps from $\mathbf{R}^{n}$ to $\mathbf{R}^{m}$. and tet 
$\|^{\prime} \subset H^{\prime}\left(\mathbf{R}^{n}, \mathbf{R}^{m}\right)$ be an open subset. Let $\delta>0$, and for $(1, x, u) \in(-\delta, \delta) \times \mathbf{R}^{n} \times \mathbb{H}^{\prime}$. let $A^{i}(t, x, u)$ be a symmetric $m \times m$ matrix, and let $B(t, x, u)$ be an m-component column vector. Suppose that $A^{i}(1, x, u)$, and $B(1, x, u)$ are $H^{3}$-functions of $(1, x)$, and ar' rational functions of $u$ with nonzero denominators. (More generally. one could " $1 s^{\prime}$ Sobolev's "condition $T$ " on compositions of $H^{2}$ functions [28].)

Given $u_{0} \in \mathbb{Z}^{3} . s>\frac{1}{2} n+2$, there is an $r:>0, r .<\delta$. and $a$ unique $u(1 . x) .|n|<1$. $x \in R^{n}$, which is $H^{z}$ in $(f, x)$ and which satisfies

$$
\begin{gathered}
u(t, x)=u_{0}(x) . \\
\partial u / \partial t=A^{\prime}(t, x, u)\left(\partial u / \partial x^{\prime}\right)+B(t, x, u) .
\end{gathered}
$$

Moreover, the solution $u(t, x)$ depends continuously on $u_{0}$ in the $H^{3}-1$ opolug!: II $A^{\prime}(t, x, u)$ and $B(t, x, u)$ are functions of $(t, x)$ in $\bigcap_{2}>n / 2+2 H^{\prime \prime}\left((-\varepsilon, \varepsilon) \times R^{\prime \prime}\right)$ and $u_{10}$ is in $\bigcap_{s>n / 2+2} H^{3}\left(\mathbf{R}^{n}\right)$, then $u(t, x)$ is in $\bigcap_{s>n / 2+2} H^{s}\left((-\varepsilon, \varepsilon) \times \mathbf{R}^{n}\right)$.

Note. For the above applications, we should replace $\partial u / \partial t$ by $A^{0}(t, x, u) i u, i t$ where $A^{0}$ is a symmetric positive-definite matrix. Here we consider the cax $s>\frac{1}{2} n+2 ;$ the case $s>\frac{1}{2} n+1$ is obtained in [14]. Moreover, 2.1 can be generalized to the case in which the coefficients and the Cauchy data satisfy asymptotic conditions. This case is more delicate and is discussed following the proof of the present theorem.

PRoor. Lel \|\|$_{s}$ denote the $H^{3}$-norm for functions $u: \mathbf{R}^{n} \rightarrow \mathbf{R}^{n}$ in $\|^{\prime}$. Let $E$ denoti the set of continuous curves $\omega:[-\delta, \delta] \rightarrow Q^{\prime}$ such that $\omega(0)=\|_{0}$ and $\|(1)(1)-\|_{11}$. $\leqq 1,-\delta \leqq 1 \leqq \delta$. Thus $E$ is a complete metric space, and we want to define a man $f: E \rightarrow E$ by

$$
f(\omega)(t)=u_{0}+\int_{0}^{1} A^{\prime}(s, x, \omega(s, x)) \frac{\partial}{\partial x^{\prime}} f(\omega)(s, x) d s+\int_{0}^{1} B(s, x,(\omega)(s, x)) d s
$$

where the integration is done as a curve in $\boldsymbol{H}^{3-1}$. From the linear theory of first order symmetric hyperbolic systems, it follows that for $\delta$ sufficiently small therc is a unique such mapping $f: E \rightarrow E$. Indeed, for $\omega \in E$, the unique solution of the linear system

$$
\begin{gathered}
u(0)=u_{0} \\
\partial u / \partial t=A^{\prime}(t, x, \omega)\left(\partial u / \partial x^{\prime}\right)+B(t, x, \omega)
\end{gathered}
$$

is exactly $f(\omega)$. Moreover, from the usual Leray estimates of the linear theory (scc Courant-Hilbert [5, p. 671]], it is easy to show that for $\omega \in E$ and for $u$ satisiying 111.

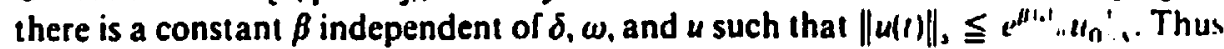
$f$ maps $E$ to $E$.

Let $F$ denote the completion of $E$ in the $H^{3-1}$-norm. We remark that $F$ is. by the Rellich-Garding theorem, a compact set, although we shall not need this fact. Next we note that for $\delta$ sufficiently small, $f: E \rightarrow E$ is a contraction in the $H^{2-1}$. norm. i.e.. for $\omega_{1}, w_{2} \in E$. there exists a $k .0<k<1$, such that 
(1)

$$
\left\|f\left(\omega_{1}\right)-f\left(\omega_{2}\right)\right\|_{1-1} \leqq k\left\|\omega_{1}-\omega_{2}\right\|_{0-1} .
$$

This follows from the estimates

$$
\begin{aligned}
\left\|f\left(\omega_{1}\right)-f\left(\omega_{2}\right)\right\|_{3-1} & \leqq \int_{0}^{1} \| A^{\prime}\left(s, x, \omega_{1}(s, x)\right) \frac{\partial}{\partial x^{\prime}} f\left(\omega_{1}\right)(s, x) \\
& \quad-A^{\prime}\left(s, x, \omega_{2}(s, x)\right) \frac{\partial}{\partial x^{\prime}} f\left(\omega_{2}\right)(s, x) \|_{2-1} d s \\
& +\int_{0}^{1}\left\|B\left(s, x, f\left(\omega_{1}\right)(s, x)\right)-B\left(s, x, f\left(\omega_{2}\right)(s, x)\right)\right\|_{2-1} d s
\end{aligned}
$$

and

$$
\begin{aligned}
& \left\|A^{\prime}\left(s, x, \omega_{1}\right) \frac{\partial}{\partial x^{i}} f\left(\omega_{1}\right)-A^{i}\left(s, x, \omega_{2}\right) \frac{\partial}{\partial x^{j}} f\left(\omega_{2}\right)\right\|_{3-1} \\
& \begin{aligned}
\leqq & \left\|A^{\prime}\left(1, x, \omega_{1}\right) \frac{\partial}{\partial x^{\prime}}\left[f\left(\omega_{1}\right)-f\left(\omega_{2}\right)\right]\right\|_{1-1} \\
& +\left\|A^{\prime}\left(t, x, \omega_{1}\right) \frac{\partial}{\partial x^{\prime}} f\left(\omega_{2}\right)-A^{\prime}\left(l, x, \omega_{2}\right) \frac{\partial}{\partial x^{\prime}} f\left(\omega_{2}\right)\right\|_{s-1} .
\end{aligned}
\end{aligned}
$$

The first term in (2) is handled as in the proof of the energy estimates; the second term is bounded by $c_{1}\left\|\omega_{1}-\omega_{2}\right\|_{3}-1\left\|f\left(\omega_{2}\right)\right\|_{1}\left(c_{1}, c_{2}, c_{3}\right.$ are constants). Thus

$$
\begin{aligned}
\sup _{\delta<\delta}\left\|f\left(\omega_{1}\right)-f\left(\omega_{2}\right)\right\|_{z-1} \leqq & c_{2} \sup _{-\delta<p_{<\delta}}\left\|f\left(\omega_{1}\right)-f\left(\omega_{2}\right)\right\|_{2-1} \\
& +\delta c_{3} \sup _{-\delta<\delta}\left\|\omega_{1}-\omega_{2}\right\|_{2-1}
\end{aligned}
$$

from which (1) follows.

Thus $f$ extends 10 a contraction on the complete metric space $F$ so by the contraction mapping principle $\int$ has a unique fixed point, a solution in $H^{2-1}$ to the quasilinear system we are studying. Since $\int$ depends continuously on $u_{0}$, so does the fixed point. We remark that the original argument of Schauder used compactness of $F$ and the Schauder fixedpoint theorem. P. Lax has pointed out that the above contraction argument can be replaced by the extraction of a weakly convergent sequence in $L_{2}$, and using $H^{3}$-boundedness, to deduce convergence in $H^{k}, k<s$. This again yields a solution in $H^{3-1}$.

Finally we show that the solution $u(\ell, x)$ in $H^{3-1}$ is in fact in $H^{3}$. The trick is to look at the differential equation satisfied by the second spatial derivative of $u$, the solution found in $\mathrm{H}^{\text {s- }}$. Now

$$
\partial u / \partial t=A^{\prime}(t, x, u)\left(\partial u / \partial x^{\prime}\right)+B(t, x, u)
$$

so if $D u$ is the first differential of $u$, 


$$
\begin{aligned}
\frac{r}{i t}(D u)= & D_{2} A^{i}(1, x, u) \frac{\vec{r} u}{\partial x^{\prime}}+D_{3} A^{\prime}(1, x, u) \cdot D u \cdot \frac{i u}{i x^{\prime}} \\
& +A^{\prime}(1, x, u) \frac{r}{r x^{i}} D u+D B(1, x, u)
\end{aligned}
$$

where $D_{2} A^{i}$ and $D_{3} A^{\prime}$ are the partial derivatives with respect to the secund and third variables. respectively. If we consider (3) as a linear equation in the unk now $n$ $r=D u$ of the form

$$
\overrightarrow{\partial t} \cdot \vec{t} t=A^{i}\left(\vec{r} t^{\prime} / x^{\prime}\right)+C \cdot t+D
$$

then we must treat $D_{3} A^{\prime}(t, x, u)\left(\partial u / \partial x^{i}\right)$ as a coefficient. However, since $u$ is only $H^{-1}$. $C^{2} u / C x^{i}$ is only $H^{-2}$. However, if we differentiate again it is easy to see that $w=D^{2} u$ satisfies

$$
\partial w / \partial l=A^{i}\left(\hat{C} w / \partial x^{i}\right)+C \cdot w+\bar{D}
$$

where now $A^{\prime}, \varnothing . \tilde{D}$ are $H^{-2}$-functions $\left(i D u / s x^{\prime}\right.$ is, for example, taken to be part of $D^{2} u$ ). The reason is just that second derivatives do not occur multiplied together as the first ones did. Now if $s>\frac{1}{2} n+3$, the coefficients are in $H^{r}, r=s-2>\frac{1}{2} n+1$. so by the linear theory $w$ which is initially in $H^{3-2}$ remains in $H^{3-2}$. Hence $u$ remains in $H^{\prime}$. (However one only needs $s>\frac{1}{2} n+2$ here by using the fact that only the lower order terms are affected and $r>\frac{1}{2} n$; see below.

This argument also shows that the map $u_{0} \rightarrow u_{1}$ is continuous in 11 and $n(1)$ merely in $H^{s-1}$. Moreover, if the coefficients $A^{\prime}(t, x, u)$ and $B(t, x, u)$ are smooth. then the same argument shows that if we have a solution in $H^{3}$ whose initial condition is in $H^{3+1}$ then in fact the solution is in $H^{s+1}$ (as long as it is defined in $H^{3}$ ). Hence smooth initial conditions remain smooth.

Now in our application, we are considering a system of the form

$$
A^{0}(1, x, u)(\partial u / \partial u)=A^{i}(1, x, u)\left(\partial u / \partial x^{i}\right)+B(1, x, u) .
$$

This case may be handled as follows. We assume as above that $A^{0} . A^{\prime}$ and $B$ are $H^{3}$-functions. By using the technique above. we are led to consider first the linear case. We proceed as in [5] to reduce to the case $A^{0}=1 \mathrm{~d}$, by writing $A^{0}=T T^{*}$ and letting $r=T^{-1} u$. However. in the case that $A^{0}$ depends on $1, x$ in an $H^{s}$ manner, this modifies the $B$ term by replacing it by an $H^{-1}$ term.

Without further conditions on the $A^{\prime}(1, x)$ Cauchy data $u_{0}$ of class $H^{\prime}$ need only have a time evolution $u(t, x)$ of class $H^{3-1}$. For example. if $A^{i}(t, x)=0$. $B(t, x)=B(x)$, then $\partial u / \partial t=B(x) u$ can be integrated explicitly 10 give $u(1, x)=e^{(8 \mid x)} u_{0}(x)$. For $B(x)$ of class $H^{3-1}, u(1, x)$ need only be of class $H^{s-1}$.

The appropriate condition on the matrices $A^{i}(t, x)$ can be found from the following standard lemma from perturbation theory [30].

2.2. Lemma. Let $F$ be a Banach space. $D_{A} \subset F$ a dense domain, and $A: D_{A} \subset F \rightarrow F$ 
a linear operator which is a generator. Let $B: F \rightarrow F$ be a bounded operator. Then $A+B: D_{A} \subset F \rightarrow F$ is a generator whose domain is exactly $D_{A}$.

Thinking of $F$ as $H^{3-1}$-functions, $D_{A}$ as $H^{3}$-functions, and $B: F \rightarrow F$ as multiplication by an $H^{s-1}$ matrix, we see that $D_{A}$ will be the domain of the closure of the operator $A=A^{i}\left(\partial / \partial x^{i}\right)$ from $H^{2}$ to $H^{p-1}$. In concrete examples like 3.1 below, the domain of this operator is not hard to work out. Thus in the symmetric hyperbolic case, where we know that $A$ is a generator, we know that solutions to the full system with a $B$ term which is $H^{-1}$ will remain in $D_{A}$ if they start out in that set.

The same remarks remain valid in the quasilinear case; that is, if $A^{\prime}$ is $H^{\prime}$ and $B$ is $H^{-1}$ then solutions which start out in $D_{A}$ will remain there. In both this and the linear case, it is important to realize that the $H^{\prime}$ energy estimates fail, and so the proof of Theorem 2.1 as given breaks down. This failure occurs because in estimating derivatives of order $s$ of the $B$ term, one runs into $H^{r}$ for $r<\frac{1}{2 n}$ and the requisite ring structure of $H^{k}$ is no longer available. However, one can use the $H^{s-1}$. estimates, and the regularity argument of Theorem 2.1 together with the linear theory Lemma 2.2. In addition to applications of Lemma 2.2 to quasilinear systems, in the next section we shall also need to consider the linear first order symmetric hyperbolic systems $A^{0}(t, x)(\partial u / \partial t)=A^{i}(t, x)\left(\partial u / \partial x^{\prime}\right)+B(t, x) \cdot u$ where $A^{0}(t, x)$ and $A^{i}(t, x)$ are of class $H^{3}$ in $(t, x)$ but $B(t, x)$ is only of class $H^{x-1}$.

3. Uniqueness for the Einstein equations. In this section we show that any two $H^{3}$-spacetimes which are Ricci flat and which have the same Cauchy data are related by an $H^{x+1}$-coordinate transformation. The key idea is to show that any $H^{3}$-spacetime when expressed in harmonic coordinates is also of class $H^{\prime}$. This in turn is based on an oid result of Sobolev [28]; namely, that solutions to the wave equation with $\left(H^{x}, H^{s-1}\right)$ coefficients preserve $\left(H^{s+1}, H^{s}\right)$ Cauchy data. We can give an easy proof of this result by using Lemma 2.2 and the well-known result that any single second order hyperbolic equation can be reduced to a system of symmetric hyperbolic equations.

3.1. TheOREM. Let $\left(\psi_{0}(x), \psi_{0}(x)\right)$ be of Sobolev class $\left(H^{3+1}, H^{3}\right)$ on $\mathbf{R}^{3}$. Then there exists a unique $\psi(t, x)$ of class $H^{3+1}$ that satisfies

$$
\begin{gathered}
(\psi(0, x), \partial \psi(0, x) / \partial t)=\left(\psi_{0}(x) . \psi_{0}(x)\right), \\
g^{\mu \nu}(t, x)\left(\partial^{2} \psi / \partial x^{\mu} \partial x^{\nu}\right)+b^{\mu}(t, x)\left(\partial \psi / \partial x^{\nu}\right)+c(t, x) \psi=0
\end{gathered}
$$

where $g^{\mu v}(t, x)$ is a Lorentz metric of class $H^{s}, b^{\mu}(t, x)$ a vector field of class $H^{3-1}$, and $c(t, x)$ is of class $H^{3-1}$.

Proof. As in the proof of Theorem 1.2, this single equation can be reduced to a first order symmetric hyperbolic system

$$
A^{0}(t, x)(\partial u / \partial t)=A^{i}(t, x)\left(\partial u / \partial x^{\prime}\right)+B(l, x) \cdot u
$$


where $A^{\prime \prime}(1, x)$ and $A^{\prime}(1, x)$ are of class $H^{3}, B(1, x)$ is of class $H^{-1}$. and $u$ is the 5 component column vector

$$
u=\left(\begin{array}{l}
\psi \\
\psi . \\
\psi .0
\end{array}\right) .
$$

In fact the $A^{0}$ and $A^{i}$ are exactly as in Theorem 1.2 with $0^{10} \rightarrow 0$ and $1^{10} \rightarrow 1$ and

$$
B(t, x)=\left(\begin{array}{ccccc}
0 & 0 & 0 & 0 & 1 \\
0 & 0 & 0 & 0 & 0 \\
0 & 0 & 0 & 0 & 0 \\
0 & 0 & 0 & 0 & 0 \\
c(t, x) & b^{\prime}(t, x) & b^{2}(t, x) & b^{3}(t, x) & b^{0}(t, x)
\end{array}\right)
$$

Regard $A=A^{\prime} \partial / 2 x^{\prime}$ as a densely defined operator on $H^{x-1}$ with domain $H^{\prime}$. A simple check using positive-definiteness of $g_{i}$, (ellipticity of the operator $\left.g^{i j}\left(l^{\lambda 2} / \hat{c} x^{i} \partial x^{j}\right)\right)$ shows that the conditions of Lemma 2.2 on the $A^{\prime}$ are met with the domain of $\bar{A}=\operatorname{cl}\left(A^{\prime} \lambda / \hat{C} x^{\prime}\right)$ in $H^{s-1}$ being at least as large as $H^{s-1} \oplus H^{\prime} \oplus H^{\prime}$ ' on the three blocks of $u$. Thus if $u_{0}$ is in $D_{A}$, then

$$
u=\left(\begin{array}{l}
\psi \\
\psi_{. i} \\
\psi_{.0}
\end{array}\right)
$$

remains in $D_{A}$, which means that $\psi$ is $H^{3+1}$ in $x$ and $H^{3}$ in $t$. From the differential equation itself we see that in fact $\psi$ is also $H^{++1}$ in $t$.

We remark that this proof "works" because we use the symmetric hyperbolic system in $u$ and thus the coefficients need only be of class $H^{s}, H^{s-1}$.

From Theorem 3.1, we can now prove that when one transforms an $H^{\text {s.space. }}$ time to harmonic coordinates, it stays $H^{3}$.

3.2. TheOREM. Let $g_{\mu v}(t, x)$ be an $H^{3}$-spacetime. Then there exists an $H^{2+1}$. coordinate transformation $\bar{x}^{\lambda}\left(x^{4}\right)$ such that

$$
\bar{g}_{\mu v}\left(\bar{x}^{\lambda}\right)=\frac{\partial x^{2}}{\partial \bar{x}^{\mu}}\left(\bar{x}^{2}\right) \frac{\partial x^{\beta}}{\partial \bar{x}^{2}}\left(\bar{x}^{\lambda}\right) g_{a \beta}\left(x^{\mu}\left(\bar{x}^{\lambda}\right)\right)
$$

is an $H^{s}$-spacetime with $\Gamma^{\mu}(\bar{i}, \bar{x})=\bar{g}^{\Delta \Delta} \Gamma_{2 \beta}^{\mu}(\bar{f}, \bar{x})=0$.

Proof. To find $\bar{x}^{\lambda}\left(x^{\mu}\right)$ consider the wave equation

$$
\square \psi=-g^{x \beta}\left(\partial^{2} \psi / \partial x^{x} \partial x^{\beta}\right)+g^{\alpha \beta} \Gamma_{2 \beta}^{\mu}\left(\partial \psi / \partial x^{\mu}\right)=0 .
$$


and let $\dot{r}(t, x)$ be the unique solution of the wave equation with Cauchy data $t(0, x)=0, \partial i(0, x) / \partial t=1$, and let $\vec{x}^{\prime}(t, x)$ be the unique solution of the wave equation with Cauchy data

$$
\bar{x}^{i}(0, x)=x^{i}, \quad \partial \bar{x}^{i}(0, x) / \partial t=0 .
$$

For $g_{\mu \nu}$ of class $H^{3} . \Gamma^{\mu}$ is of class $H^{2-1}$, so by Theorem $3.1, \bar{i}(t, x)$ and $\bar{x}(1, x)$ are $H^{s+1}$-functions and in fact by the inverse function theorem for $H^{\prime}$-functions, $(\tilde{f}(t, x), \bar{x}(t, x))$ is an $H^{+1}$ diffeomorphism in a neighborhood of $t=0$.

Since $\square \bar{x}^{\mu}(t, x)=0$ is an invariant equation,

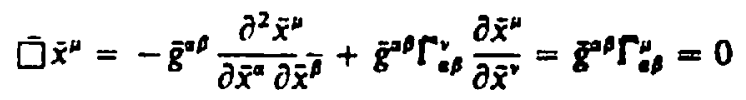

in the barred coordinate system, so $\bar{x}^{\mu}$ is a system of harmonic coordinates.

As a simple consequence of Theorem 3.2 we have the following uniqueness result for the Einstein equations:

3.3. THEOREM. Let $g_{u v}(1, x)$ and $\bar{g}_{u v}(1, x)$ be two $H^{3}$-spacetimes with zero Ricci tensor and such that $\left(g_{\mu v}(0, x), \partial g_{\mu v}(0, x) / \partial t\right)=\left(\bar{g}_{\mu v}(0, x), \partial \bar{g}_{\mu v}(0, x) / \partial t\right)$. Then $g_{\mu v}(t, x)$ and $\overline{\mathrm{g}}_{\mu \mathrm{v}}(t, x)$ are related by an $H^{3+1}$-coordinate change in a neighborhood of $t=0$.

Proof. From Theorem 3.2 there exist $\mathrm{H}^{3+1}$-coordinate transformations $y^{\mu}\left(x^{3}\right)$ and $\bar{y}^{\mu}\left(x^{\alpha}\right)$ such that the transformed metrics $\left(\partial x^{p} / \partial y^{\mu}\right)\left(\partial x^{p} / \partial y^{\nu}\right) g_{x g}$ and $\left(\partial x^{\alpha} / \partial \bar{y}^{\mu}\right)\left(\partial x^{\beta} / \partial \bar{y}^{\nu}\right) \bar{g}_{\alpha \beta}$ satisfy $R_{\mu \nu}^{(h)}=0$. Since the Cauchy data for $g_{\mu v}$ and $\bar{g}_{\mu v}$ are equal the transformed metrics also have the same Cauchy data. By the uniqueness part of Theorem 1.2 $\left(\partial x^{\alpha} / \partial y^{\mu}\right)\left(\partial x^{\beta} / \partial y^{\prime \prime}\right) g_{\alpha \beta}=\left(\partial x^{0} / \partial \bar{y}^{\prime \prime}\right)\left(\partial x^{\beta} / \partial \bar{y}^{\prime}\right) \bar{g}_{\alpha \beta}$. Since the composition of $H^{s+1}$-coordinate changes is also $H^{s+1}, \bar{g}_{o p}$ is related to $g_{x \neq}$ by an $H^{s+1}$-coordinate change in a neighborhood of $t=0$.

4. The Einstein system on the manifold $\mathscr{H}$. We now consider a dynamical formulation of general relativity from the 3-dimensional point of view of Arnowitt. Deser and Misner [1], DeWitt [6], and Wheeler [29]. All tensor fields, such as g, $k, X$ are referred to a fixed oriented smooth 3-dimensional manifold $M$.

$$
\begin{aligned}
& \text { Let } \\
& \mathscr{H}=\operatorname{Riem}(M)=\text { manifold of all smooth Riemannian metrics (positive- }
\end{aligned}
$$
$S_{\mathbf{2}}(M)=$ the linear space of all smooth symmetric 2-covariant tensor fields on $M$; and

In the dynamical formulation of general relativity, one is concerned with the evolution of initial Cauchy data $(\xi, h) \in M \times S_{2}(M)$ on some 3-dimensional hypersurface $M$ of a yet to be constructed Ricci-flat (vacuum) spacetime $V_{4}$. 
As one is interested in finding the evolution g, of Riemannian metrics only up to the isometry class $\left\{\left(\eta_{1}^{-1}\right)^{*} g_{1} \mid \eta_{1} \in \mathscr{C}\right\}$ of $g_{1}$ (here $\left(\eta_{1}^{-1}\right)^{*}$ is the "push forward" of covariant tensor fields). the evolution is determined only up to an arbitrary curve $\eta_{1} \in J$ with $\eta_{0}=$ the identity diffeomorphism. In other words, only the orbit class or geometry of $g_{1}$ is determined (see DeWill [6] and Fischer [11] for the structure of the orbit space $\mathscr{H} / \mathcal{S})$. Moreover, one is free to specify on $M$ an arbitrary system of clock rates.

These degeneracies are reflected in the evolution equations as follows:

The EINSTEIn SYSTEM. Let $(\xi, k) \in \mathscr{A} \times S_{2}(M)$ satisfy the constraints:

$$
\left.\delta(k-\xi(\operatorname{Tr} \hat{k}))=0, \quad \frac{1}{2}(\operatorname{Tr} \hat{k})^{2}-\hat{k} \cdot \hat{k}\right)+2 R(\dot{g})=0 .
$$

Let $X_{1}$ be an arbitrary time-dependent vector field on $M$ (the shift vector held) und $N$, an arhitrary time-dependent scalar field on $M$ (the lapse function) such thut

$$
\begin{aligned}
N_{i}(m) & >0 . \\
N_{i}^{2}-\left\|X_{0}\right\|^{2} & >0 \quad \text { for all }(1, m) \in R \times M .
\end{aligned}
$$

The problem is to find a curve $\left(g_{1}, k_{1}\right) \in . M \times S_{2}(M)$ which satisfies the ecolurmm equations

$$
\begin{aligned}
& \partial g_{1} / \partial t=N_{1} k_{1}-L_{x_{1}} g_{1}, \\
& \partial k_{1} / \partial t=N_{1} S_{l}\left(k_{1}\right)-2 N_{1} \operatorname{Rid}\left(g_{1}\right)+2 \operatorname{Hess}\left(N_{1}\right)-L_{x_{1}} k_{1} .
\end{aligned}
$$

and which has initial conditions $\left(g_{0}, k_{0}\right)=\left(k_{.} k\right)$.

Our notation is the following:

$\delta k=$ divergence of $k=(\delta k)_{1}=-k_{i j}^{i}$.

$\operatorname{Tr} k=$ Trace $k=g^{i j} k_{i j}=k_{i}^{i}$.

$k \cdot k=$ dot product for symmetric tensors $=k_{i j} k^{\prime j}$.

$k \times k=$ cross product for symmetric tensors $=k_{i j} k_{j}^{\prime}$,

$S_{p}(k)=k \times k-\frac{1}{2}(\operatorname{Tr} k) k=k_{i} k_{j}^{\prime}-\frac{1}{2}\left(g^{m n} k_{m n}\right) k_{i j}$.

$\|X\|^{2}=$ norm of $X=g_{i j} X^{\prime} X^{j}$.

$L_{X} g_{1}=\left\{\right.$ Lie derivative of $g$ with respect to the time-dependent vector ficld $X_{1} ;$

$=X_{i j j}+X_{i j} l_{i j}=$ covariant derivative with respect to the time-dependent metric).

$L_{x_{1}} k_{1}=$ Lie derivative of $k_{1}=X^{\prime} k_{1 \mid 11}+k_{i j} X^{\prime}{ }_{j j}+k_{j 1} X^{\prime}{ }_{\mid i}$,

$\operatorname{Ric}\left(g_{1}\right)=\left(\right.$ Ricci curvature tensor formed from $\left.g_{1}\right)=R_{1 j}=\Gamma_{1, k}^{k}-\Gamma_{k_{1}, j}^{k}+$ $\Gamma_{i j}^{k} \Gamma_{k 1}^{\prime}-\Gamma_{i k}^{i} \Gamma_{i,}^{k}$.

$R\left(g_{1}\right)=$ scalar curvature $=R_{k}^{k}$,

Hess $(N)=$ Hessian of $N=$ double covariant derivative $=N_{1 \mid 1,}$.

In the case that we choose $N_{1}=1$ and $X_{1}=0$, the proper configuration space for the Einstein system is the manifold. $\mathscr{K}$. We equip. $\mathscr{H}$ with a metric $\mathscr{S}$. referred to as the Dellitt metric, by setting for $g \in \mathscr{A}$. 


$$
\begin{gathered}
\mathscr{G}_{R}: T_{R} \mathscr{H} \times T_{R}-\mathscr{H} \approx S_{2}(M) \times S_{2}(M) \rightarrow \mathbf{R} . \\
\mathscr{G}_{R}\left(h_{0} k\right)=\int_{M}((\operatorname{Tr} h)(\operatorname{Tr} k)-h \cdot k) \mu_{z}
\end{gathered}
$$

where $\mu_{x}=(\operatorname{det} g)^{8 / 2} d x^{1} \wedge d x^{2} \wedge d x^{3}$ is the usual volume element.

The following is a straightforward computation (see [12]):

4.1. Propositnon. The Lagrangian $L_{0}(g, h)=\frac{1}{2} \mathscr{G}_{g}(h . h)$ is nondegenerate and the associated Lagrangian vector field exists and is given by the second order system

$$
\begin{aligned}
& \partial g_{1} / \partial t=k_{1} . \\
& \partial k_{1} / \partial t=k_{t} \times k_{1}-\frac{t}{t}\left(\operatorname{Tr} k_{t}\right) k_{t}-\frac{1}{b}\left(\left(\left(\operatorname{Tr} k_{1}\right)^{2}-k_{1} \cdot k_{1}\right) g\right) .
\end{aligned}
$$

For each $(\xi, k) \in \mathscr{H} \times S_{2}(M)$ ihere exisis a unique smooth curoe $\left(g_{1}, k_{1}\right) \in \mathscr{A} \times S_{2}(M)$ defined for short time with initial conditions $\left(g_{0}, k_{0}\right)=\left(\xi_{,} k\right)$ and which satisfies $(Z)$.

Now one adds a potential term to $L_{0}$; set

$$
L(g, k)=\frac{1}{2} \mathscr{G}_{\imath}(k, k)-2 \int_{M} R(g) \mu_{u}
$$

where $R(g)$ is the scalar curvature of $g$. Adding this potential term adds a gradient term to the equations of motion. For the potential $V=2 \int_{M} N R(g) \mu_{\text {g }}$ (where $N$ is a positive scalar on $M$ included for later use), a computation gives

$$
-\operatorname{grad} V=-2 N\left(\operatorname{Ric}(g)-\frac{1}{4} R(g) g\right)+2 \operatorname{Hess}(N) \text {. }
$$

where the gradient has been computed with respect to the DeWitt metric. Using the pointwise conservation law $\left.\frac{f}{2}(\operatorname{Tr} k)^{2}-k \cdot k\right)+2 R(g)=0$ (see [12]). we have

4.2. Proposition. $\left(g_{1}, k_{1}\right) \in \mathscr{H} \times S_{2}(M)$ is an integral curve of the second order system determined by $L=\frac{1}{2} \mathscr{G}_{R}(k, k)-2 \int_{M} R(g) \mu_{R} i f f$

$$
\begin{aligned}
& \partial g_{1} / \partial t=k_{1} . \\
& \partial g_{1} / \partial t=S_{t_{1}}\left(k_{l}\right)-2 \operatorname{Rid}\left(g_{l}\right) .
\end{aligned}
$$

Eardley, Liang, and Sachs [8] have given conditions for which the velocity terms $S_{g}(k)$ dominate the $R i c(g)$ term (for example near a singular hypersurface) so that the latter can with some justification be neglected. In this case the integral curves can be given explicitly, and are the geodesics of $\mathscr{A}$ with respect to the metric $\mathcal{G}$.

5. The evolution equations with a shift vector field and space-body transitions. Now suppose we consider the equations with an arbitrary shift vector field $X_{1}$. We assert that there is a simple method for solving these equations if the solution for $X=0$ is known.

5.1. Proposition. Let $g_{1}, k_{1}$, he a solution of the Einstein system with $N=1, X=0$. 
Then giren $X$, we construct it flow' $\eta_{1}$. Then the solution of the Einstein system with $N=1$, shiff $X_{1}$, and the same initial conditions $8_{0}, k_{0}$ is given by

$$
\tilde{\mathbf{g}}_{t}=\left(\eta_{1}^{-1}\right)^{*} \xi_{1}, \quad k_{t}=\left(\eta_{t}^{-1}\right)^{*} k_{1} .
$$

ProOf. The extra terms involving the Lie derivatives are picked up as follows:

$$
\begin{aligned}
& \partial \bar{k}_{1} / \partial t=\left(\eta_{1}^{-1}\right)^{*}\left(\partial k_{1} / \partial \imath\right)-L_{x_{1}}\left(\eta_{1}^{-i}\right)^{* k_{1}} \\
& =\left(\eta_{1}^{-1}\right)^{*}\left(S_{z_{1}}\left(k_{1}\right)-2 \operatorname{Ric}\left(g_{1}\right)\right)-L_{x_{1}} k_{1} \\
& =S_{\bar{i}_{1}}\left(k_{1}\right)-2 \operatorname{Rid}\left(\bar{g}_{1}\right)-L_{x_{1}} k_{1} .
\end{aligned}
$$

where we have used the fact that $\partial\left(\eta_{1}^{-1}\right)^{*} k / \partial t=-L_{x_{1}}\left(\eta_{1}^{-1}\right)^{*} k$. 圆

Proposition 5.1 shows that even though the evolution equations with a shift involve extra terms which are nonlinear and involve derivatives, the more general system can be solved merely by solving an ordinary differential equation: namely. by finding the flow of $X_{1}$.

In order to take into account the shift veclor field $X$, we enlarge the configuration space $\mathscr{N}$ to $\mathscr{T} \times \mathcal{H}$. For $\eta \in \mathscr{O}$, it is easy to see that $T_{\eta} \mathscr{V}$ is the set of maps $X \cdots \eta$ where $X$ is a vector field on $M$. The Lagrangian of the preceding section is transferred to $U \times . M$ by setting, for $(\eta, g) \in \mathcal{S} \times . H$,

$$
\begin{aligned}
& L: T_{\eta} \mathscr{Q} \times T_{2} \cdot H \rightarrow \mathbf{R}, \\
& \quad(X \circ \eta, h) \rightarrow \frac{1}{2} \mathscr{G}_{2}\left(h+L_{x} g_{1} h+L_{x} g\right)-2 \int_{M} R(g) \mu_{2} .
\end{aligned}
$$

We observe that for $\lambda \in \mathbf{R}, \lambda \neq 0, L(\lambda X \circ \eta, \lambda h)=\lambda^{2} L(X \circ \eta, h)$ so that $L$ is quadratic in the velocities $(X \circ \eta, h)$. On $T, \mu$, of course, this is not true.

On $T(G \times . \mathscr{H}), E$ is, roughly speaking, degenerate in the direction of $\%$. This degeneracy has the effect of introducing some ambiguity into the equations of motion, which is, however, precisely removed by the specification of a curve $\eta_{1} \in \mathscr{Q}$. A direct computation proves the following:

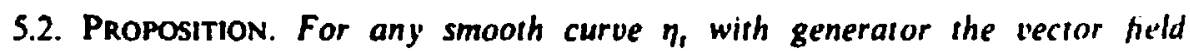
$X_{1}=\left(d \eta_{:} / d t\right) \cup \eta_{l}^{-1}$, a possible Lagrangian vector field for the de'generate Lagrangian $L(\eta . g: X \cdot \eta \cdot h)=L\left(g . h+L_{x} g\right)$ is given by the equations

$$
\begin{aligned}
& \partial g_{t} / \partial t=k_{1}-L_{x_{1}} g_{1}, \\
& \partial k_{t} / \partial t=S_{t_{1}}\left(k_{t}\right)-2 \operatorname{Ric}\left(g_{1}\right)-L_{x_{1}} k_{t} .
\end{aligned}
$$

There is a natural action of the group $\mathscr{S}$ on $\mathscr{Q} \times . \|$ given by

$$
\begin{gathered}
\Phi_{\eta}: Q \times A \rightarrow Q \times \mathscr{H} \\
(\zeta . g) \rightarrow\left(\eta \circ \zeta \cdot\left(\eta^{-1}\right)^{*} g\right) .
\end{gathered}
$$


This action leads to a natural symmetry and consequent conservation laws for our system.

5.3. Proposition. Let $\Phi_{n}: \Omega \times \mathscr{N} \rightarrow \Omega \times \mathscr{H}$ be as above with tangent action $T \Phi_{n}: T(\mathscr{A} \times \mathscr{A}) \rightarrow T(S \times \mathscr{A})$. Then $L \circ T \Phi_{\eta}=L$ for each $n \in \mathscr{P}$ and $\delta(k-g(\operatorname{Tr} k))$ $\otimes \mu_{g}(\otimes=$ tensor product $)$ is a constant of the motion.

We remark that the standard conservation theorems (c. [22]. [23]), used to prove this proposition, have to be modified to take into account the degeneracy of $L$. The infinite dimensionality of the symmetry group leads to a differential rather than an integral identity; see [12] for details.

The Lie derivative terms that appear in 5.2 have a natural geometric interpretation related to changing from space to body coordinates in a munner similar to that of the rigid body and hydrodynamics (cl. Marsden-Abraham [23] and EbinMarsden [9]). More specifically we consider the manifold $M$ to be the body, and the flow' $\eta_{1}$ of the shif vector field $X$, as being a rotation of $M$. We then make the convention that an observer is in body coordinates if he is on the manifold, and is in space coordinates if he is off the manifold.

Now let $g$, be a time-dependent metric field on $M$. We assume that the field is rigidly attached $10 M$ as it moves so that we set $g_{1}=g_{000 y}$. An observer in body

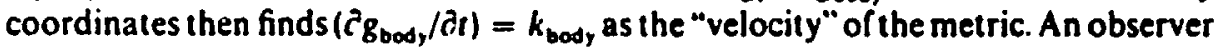
in space coordinates sees the metric field $g_{\text {body }}$ as it is dragged past him by the mov. ing manifold: he sees the metric field $g_{\text {space }}=\left(\eta_{1}^{-1}\right)^{*} g_{\text {body }}$ and computes

(I) $\partial g_{\text {,pace }} / \overrightarrow{a l}=k_{\text {space }}-L_{x} \boldsymbol{g}_{\text {space. }}$.

(2) $\partial k_{\text {space }} / \partial t=S_{\text {sope. }}\left(k_{\text {spase }}\right)-2 \operatorname{Ria}\left(g_{\text {space }}\right)-L_{x} k_{\text {space }}$,

where $k_{\text {space }}=\left(\eta_{1}^{-1}\right)^{*} k_{\text {body }}$. But (1) and (2) are just the evolution equations (with $N_{1}=11$.

Finally. we remark that the Hessian term in the evolution equations can be accounted for by introducing the general relativistic time translation group $F=C^{x}(M: R)$ and defining an extended Lagrangian on $\mathscr{F} \times \mathscr{Q} \times A$. The pointwise conservation of the Hamiltonian is closely related to the invariance of the Lagrangian under the action of the group of general relativistic time translations $\mathscr{F}$. Moreover, as with the shift vector field, there is associated with an arbitrary lapse function and a solution of the evolution equations with $N=1$ and with given Cauchy data a proper time function $\tau$ and an intrinsic shift vector field $Y$. From the integration of $\gamma$, together with $\tau$, we determine from the solution for $N=1$ the solution with the arbitrary lapse function and with the same Cauchy data See [12] for a detailed analysis of these topics.

\section{BiBLIOGRAPHY}

1. R. Arnowitt. S. Deser and C. W. Misner. The dynamies of general relativity, Gravitation: An Introduction to Current Research. Wiley. New York. 1962. MR $26 \neq 1182$. 


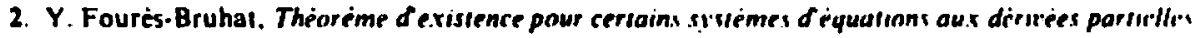
non linéaires. Acla Math. 88 (1952). 141-225. MR 14. 756.

3. Y. Choquet-Bruhal. Espaces-lemps einsterniens generour chocs grartossonnels, Ann. Inst H Poincaré Sect. A 8 (1968). 327-33R. MR 38 \% 1897.

4. - Solutions $C^{\prime}$ dequastons hypesholiques non limeaires, C. R. Acad. Sct Paris 272 (197)। $386-388$.

5. R. Courant and D. Hilbert. Methods of mashemasical physics. Vot. II: Parsial differential eqmes. tions (Vol. II by R. Courant). Interscience. New York. 1962. MR 25 \#4216.

6. B. DeWitt. Quantum theory of gravily. I. The cononiral thenr). Phys. Rev. 160 (1967). 1113 114x

7. P. A. Dionne. Sur les prohlemes de Cauchy hyperholiques hien posés. I. Analyse Math. 10 (1962 h1, 1.90. MR $27 \approx 472$

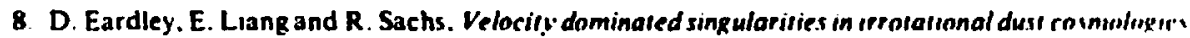
J. Mathematical Phys. 13 (1972). 99. 107.

9. D. G. Ebin and J. Marsden, Groups of diffeumorphisms and the molion of an incampresuthle flutud Ann. of Math. (2) 92 (1970), 102-163. MR $42 \% 6865$.

10. A. Einstein. Nüherungsweise Iniegration der Feldgleichungen der Grathatinn. S.B Privus Akad. Wiss. 1916. 688-696.

II. A. Fischer, "The theory of superspace," in Relotivity. M. Carmeli. S. Fickler and L. Willitn (Editors). Plenum Press. New York. 1970. 303-357.

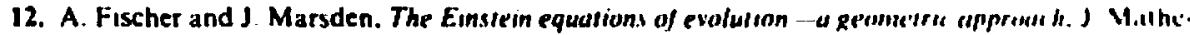
malical Phys. 13 (1972). 546-568.

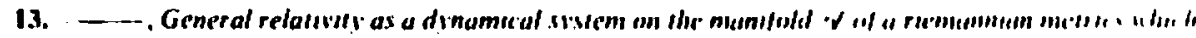

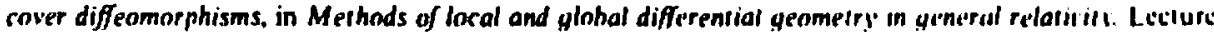
Noles in Physics, vol. 14, Springer-Verlag, Berlin, 1972. 176-188.

14. —- The Einstein evolution equations as a first-order quasi-lintar symmetric huperhesier swe'tu 1. Comm. Math. Phys. 28 (1972), 1-38.

15. —_. First -order symmetric hyperbolic systems on non-compact manitolds and gentral retari!.", (lo appear).

16. F. Frankl. Üher das Anfongsweriproblem für lineare und nichilineare hyperholische partie'le Differentialgleichungen zweirer Ordnung, Mat. Sb. 2 (44) (1937), 814-868.

17. K. O. Friedrichs. Summeiric hyperholis linear differential equolums. Comm Pure Appl. Malh 7 (1954), 345-392. MR 16, 44.

18. C. Lanczos, Ein vereinfachendes Koordinatensystem für die Einsteinschen Gratilationsgleirhungen. Phys. Z. 23 (1922). 537-539.

19. P. Lax. On Couchy's problem for hyperbolic equotions and the differentiabiliny of solutions of elliptic equations, Comm. Pure Appl. Math. 8 (1955). 615-633. MR 17, 1212

20. J. Leray. Leclures on hyperbolic equations with variable coefficients, Institute for Advanced Studies. Princeton. N.J., 1952.

21. A. Lichnerowicz. Relativisuic hydrodynamics and magnerohydrodinamics. Benjamin. New York. 1967.

22. J. E. Marsden. Hamillanian one parameter groups: A mathematical exposition of infinile dimensional Hamilionian sistems with applications in classical and quantum mechanirs. Arct. Rational Mech Anal. 28 (1967,68), 362-396. MR $37=1735$

23. J. Marsden and R. Abraham. Hamilianian mechanics on Lie groups and hydrodynamics, Proc. Sympos. Pure Math., vol. 16. Amer. Math. Soc., Providence, R.I., 1968, pp. 237-244

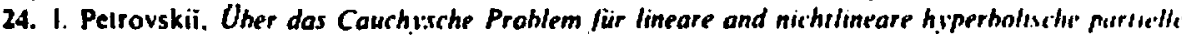
Differentialgleichunges. Mat. Sb. 2 (4) (1937), 814-868.

25. J. Schauder. Das Anfangswertproblem einer quasilinearen hyperbolischen Differentialgleichungen weiter Ordnung in beliebiger Anzahl von unabhangigen Verönderlichen. Fund. Math. 24 (1935). 213-246

26. S. S. Sobolev. Meihode noulelle à sésourdre le probleme de Cauchy pour les èquations lineaires 
h.perholiques normulex. Mat. Sb. 1 (43) (1936). 39-72.

27. -_- Sur ta théorie des équations hyperboliques aux diriveies portielles, Mal. Sb. $S$ (47) (1939). 71.-99. (Russian) MR 1. 237.

28. - - Applications of functional analysis in mathematical physics, Izdal. Leningrad. Gos. Univ., Leningrad. 1950: English transl., Transl. Math. Monographs, vol. 7. Amer. Math. Soc., Providence. R.J.. 1963. MR 14. 565: MR $29 * 2624$.

29. I A. Wheeier, Geometrodynamics and the issue of the final state. Relativilc, Groupes et Topologie (Lectures. Les Houches, 1963 Summer School of Theoret. Phys., Univ. Grenoble), Gordon and Breitch, New York, 1964, pp. 315-520. MR 29 iw 5596.

30. K. Yosida, Funcsional analysis, Die Grundlehren der math. Wissenschaften, Band 123, Academic Press. New York: Springer-Verlag. Berlin, 1965. MR 31 \# 5054.

Univiasit Y or Calitorknia. SANta Criz and Berkiley 